\title{
RACK1 modulates apoptosis induced by sorafenib in HCC cells by interfering with the IRE1/XBP1 axis
}

\author{
TI ZHOU ${ }^{1}$, XING LV ${ }^{1}$, XIN GUO $^{1}$, BAI RUAN ${ }^{1}$, DONG LIU ${ }^{1}$, RUI DING ${ }^{1}$,

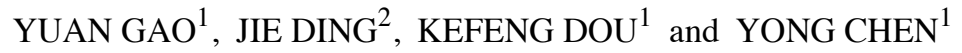 \\ ${ }^{1}$ Department of Hepatobiliary Surgery, Xijing Hospital, Fourth Military Medical University, Xi'an, Shaanxi 710032; \\ ${ }^{2}$ Department of Pharmacy, Xi'an Central Hospital, Xi'an, Shaanxi 710032, P.R. China
}

Received December 27, 2014; Accepted March 24, 2015

DOI: $10.3892 /$ or.2015.3920

\begin{abstract}
Sorafenib is one of the preferred drugs for the treatment of advanced primary hepatocellular carcinoma (HCC). However, its side-effects and acquired resistance limit its use. The unfolded protein response (UPR) induced by chemotherapeutics has been demonstrated to be required for tumor cells to maintain malignancy and therapy resistance. Activation of the IRE1/XBP1 pathway during the UPR is important for tumor survival under pathophysiological conditions. In the present study, we found that the UPR was activated and RACK1 was overexpressed in three human HCC cell lines and in HCC samples. Activation of the IRE1/XBP1 signaling pathway plays a protective role when HCC cells encounter endoplasmic reticulum (ER) stress due to in vitro sorafenib treatment. We then found that the interaction between IRE1 and RACK1 was essential for the activation of IRE1 signaling in sorafenibtreated cells. Exogenous overexpression of RACK1 enhanced the phosphorylation level of IRE1 and increased XBP1 mRNA splicing activity, which protected the HCC cells from sorafenib-induced apoptosis. However, the re-expression of RACK1 led HCC cells to regain susceptibility to sorafenibinduced apoptosis. Taken together, the present study suggests that the RACK1/IRE1 complex may contribute to activation of the UPR in HCC cells. Targeting RACK1 in combination with sorafenib administration is a potential strategy for clinical trials of advanced HCC treatment.
\end{abstract}

Correspondence to: Professor Yong Chen or Professor Kefeng Dou, Department of Hepatobiliary Surgery, Xijing Hospital, Fourth Military Medical University, Xi'an, Shaanxi 710032, P.R. China

E-mail: yongchen1962@gmail.com

E-mail: doukf_fmmu@foxmail.com

Abbreviations: HCC, hepatocellular carcinoma; ER, endoplasmic reticulum; UPR, unfolded protein response; XBP-1, X-box binding protein 1; TM, tunicamycin; ERAD, ER associated degradation; GRP78, 78-kDa glucose-regulated protein; IRE1, inositol-requiring enzyme 1; RACK1, receptor for activated C kinase 1

Key words: hepatocellular carcinoma, chemotherapy, unfolded protein response, inositol-requiring enzyme 1, receptor for activated C kinase $1, \mathrm{X}$-box binding protein 1

\section{Introduction}

Hepatocellular carcinoma (HCC) is one of the most common malignancies worldwide and a common cause of cancerrelated death globally (1). Unfortunately, the prognosis, early diagnosis and treatment of $\mathrm{HCC}$ remain poor. Few patients diagnosed with HCC are eligible for curative therapies, including surgical resection, percutaneous ablation and liver transplantation. Most patients are diagnosed at a late stage of the disease when potentially curative therapies are least effective $(2,3)$. Therefore, new therapies are urgently needed for these patients.

Sorafenib, an oral multikinase inhibitor of several tyrosine protein kinases and Raf kinases, induces apoptosis in human leukemia and other malignant cells, and is currently the only drug categorized as a preferred treatment for advanced primary HCC $(4,5)$. Investigators have reported that sorafenib inhibits tumor cell proliferation and promotes apoptosis by inhibiting several kinases in the mitogen-activated protein kinase (MAPK) pathway and the translation of pro-survival factors, such as Mcl-1 and Bcl-2 (6-8). Clinical studies have confirmed the efficacy of sorafenib during HCC treatment; however, the drug has several side-effects, and resistance to sorafenib is increasing. Therefore, it is necessary to clarify the mechanisms of sorafenib chemoresistance and to identify other therapeutic biomarkers that might be combined with sorafenib to improve its efficacy.

Cancer cells commonly encounter endoplasmic reticulum (ER) stress during tumor initiation and progression due to hypoxia, glucose deprivation, DNA damage or ER calcium depletion (9). The unfolded protein response (UPR) activated during ER stress is a compensatory cellular defense mechanism and an adaptive response that allows tumor cells to survive under these pathophysiological conditions (10). The UPR in response to chemotherapeutics is necessary for tumor cells to maintain malignancy and therapy resistance (11). Identification of the UPR components that are activated or suppressed in tumors and investigation of the potential of cancer therapeutics by targeting the UPR may be potentially useful to inhibit tumor growth and improve cancer therapy $(12,13)$. IRE1 is the only identified ER stress sensor in yeast and is essential for the UPR in animals and plants (14). As an ER transmembrane protein, IRE1 monitors ER homeostasis through an ER 
luminal stress-sensing domain and triggers the UPR through a cytoplasmic kinase domain and an RNase domain (15). IRE1 activation and attenuation play a critical role in cell fate decisions during ER stress responses $(16,17)$. Upon ER stress, IRE1 RNase is activated through a conformational change, autophosphorylation and higher-order oligomerization. Activated IRE1 subsequently splices a 26-nucleotide sequence from the mRNA encoding X-box binding protein 1 (XBP1), resulting in the translation of activated/spliced XBP1 (XBP1s). $\mathrm{XBP} 1 \mathrm{~s}$ is a potent transcription factor and one of the key regulators of the UPR; it transduces the UPR to the nucleus and regulates many target genes, including ER chaperones, ER-associated degradation (ERAD) components and other transcription factors $(18,19)$. Recent studies have indicated that XBP1 mRNA splicing activity does not increase progressively under increasing ER stress intensity and/or duration, and is only activated during the adaptive/pro-survival phase of ER stress (20). The IRE1/XBP1 pathway is important for tumor survival under ER stress (21). The overexpression of XBP1 was found in various solid tumors, including breast cancer and HCC. Additionally, prolonged IRE1 signal activation was shown to enhance cell survival (22). Taken together, these results suggest that the IRE1/XBP1 axis may play an important role in the response of tumors to chemotherapeutics and serve as a key regulator of cell fate.

RACK1 was originally identified as an intercellular scaffold protein of the Trp-Asp (WD) repeat protein family (23). RACK1 can recruit and bind many kinases and receptors, such as PKC and DJ-1, thereby impacting a wide range of signal transduction pathways $(24,25)$. RACK1 plays an important role in multiple cellular functions, including cell growth, migration and differentiation $(26,27)$. Studies have found that RACK1 is upregulated in several tumor types, such as breast tumors and HCC $(28,29)$. Moreover, RACK1 might function as an internal factor that contributes to stress-mediated chemotherapy resistance (30). However, the regulatory mechanism of RACK1 in the stress response has remained largely elusive.

A study by Qiu et al (31) showed that RACK1 interacts with IRE1 in a glucose-stimulated or ER stress-responsive manner. This finding demonstrated that RACK1 is required for IRE1 activation under ER stress conditions in pancreatic $\beta$-cells. However, the interaction of RACK1 with IRE1 and the role of RACK1 in the IRE1 signaling pathway have not been evaluated in HCC. In the present study, we showed that RACK1 is overexpressed in both HCC tissue samples and cell lines, and RACK1 is required for IRE1 signaling activation under ER stress conditions. This interaction could contribute to the ability of HCC cells to adapt to stressful conditions. Our findings provide a new strategy to promote the chemotherapeutic effects of sorafenib by targeting the IRE1/XBP1 axis.

\section{Materials and methods}

Clinical samples. HCC and adjacent non-tumor (NT) tissue samples were obtained by surgical resection from 46 patients at our hospital between June 2011 and April 2013 (see detailed clinicopathological features in Table I). The HCC and NT tissues were verified by the classification of the General Rules for the Clinical and Pathological Study of Primary Liver Cancer (32). Resected tissues were frozen immediately at
Table I. Summary of the clinicopathological variables.

\begin{tabular}{lc}
\hline Characteristics & Data \\
\hline Patients & 46 \\
Gender & \\
Male & 40 \\
Female & 6 \\
Age (years), range; median & $37-71 ; 49.7$ \\
Tumor size (cm), range; median & $0.6-15 ; 5.2$ \\
Clinical stage, $\mathrm{n}$ & \\
I & 2 \\
II & 14 \\
III & 30 \\
IV & 0 \\
\hline
\end{tabular}

$-80^{\circ} \mathrm{C}$ or fixed in $10 \%$ formalin. Informed consent was obtained from all patients or their guardians for subsequent use of their resected tissues, and the Ethics Committee of Xijing Hospital, The Fourth Military Medical University, approved all aspects of the present study.

Plasmid construction and small-interfering RNAs (siRNAs). To construct pCDNA3.1(+)-RACK1, the coding sequence of human RACK1 was amplified by PCR and digested with HindIII and EcoRI. The RACK1 cDNA was ligated into a similarly digested pCDNA3.1(+) vector. IRE1 siRNA (sc-40705), RACK1 siRNA (sc-36354) and the non-targeting control siRNA (sc-37007) were purchased from Santa Cruz Biotechnology (Santa Cruz, CA, USA).

Cell culture and treatment. The human HCC cell lines HepG2, SMCC7721 and MHCC97 were maintained in our laboratory and cultured in Dulbecco's modified Eagle's medium (DMEM) (HyClone, Logan, UT, USA) supplemented with 10\% heatinactivated fetal calf serum (Gibco, Grand Island, NY, USA), $100 \mathrm{U} / \mathrm{ml}$ penicillin $\mathrm{G}$ and $100 \mu \mathrm{g} / \mathrm{ml}$ streptomycin at $37^{\circ} \mathrm{C}$ in a humidified atmosphere containing $5 \% \mathrm{CO}_{2}$. Aliquots of HepG2 or MHCC97 cells were transfected with Lipofectamine 2000 (Invitrogen, Carlsbad, CA, USA) premixed with siRNA or a scrambled sequence, following the manufacturer's instructions. Successfully transfected cells were obtained after $48 \mathrm{~h}$ and analyzed via western blotting.

Protein extraction and western blot analysis. Tissues and cells were lysed in RIPA lysis buffer (20 mM Tris- $\mathrm{HCl}, \mathrm{pH} 7.5$, $100 \mathrm{mM} \mathrm{KCl}, 0.1 \%$ Nonidet P-40, 1 mM EDTA and $10 \%$ glycerol) containing $1 \mathrm{mM}$ PMSF, $1 \%$ protease inhibitor cocktail (Sigma, St. Louis, MO, USA) and 1\% phosphatase inhibitor cocktail I/II (Sigma) for $20 \mathrm{~min}$ at $4^{\circ} \mathrm{C}$. Then, the total protein in the lysates was determined using a bicinchoninic acid (BCA) assay. Equal amounts of samples were mixed with Laemmli sample buffer and placed in a boiling waterbath for $5 \mathrm{~min}$. Proteins were then separated by $10 \%$ sodium dodecyl sulfate-polyacrylamide gel electrophoresis (SDS-PAGE) and transferred to nitrocellulose membranes (Amersham 
Biosciences, Uppsala, Sweden). Then, the membranes were incubated with specific primary antibodies for GRP78 (Bip), IRE1, pT-724 IRE1, RACK1 and XBP1s (Abcam, Cambridge, MA, USA) overnight at $4^{\circ} \mathrm{C}$. Incubation with horseradish peroxidase (HRP)-coupled secondary antibodies (ProteinTech Group, Chicago, IL, USA) was performed for $1 \mathrm{~h}$ at room temperature. Signals were detected using enhanced chemiluminescence (Thermo Fisher Scientific Inc., Rockford, IL, USA) and scanned with a gel imaging system (Bio-Rad Laboratories, Berkeley, CA, USA). The monoclonal antibodies anti-GAPDH (Kangcheng, Shanghai, China) or anti- $\beta$-actin (Sigma) were used as controls.

Immunohistochemistry. Immunohistochemical staining for RACK1 was performed on formalin-fixed, paraffin-embedded specimens using the streptavidin-peroxidase (SP) method. Histological slides, 4-mm in thickness, were baked in an oven at $60^{\circ} \mathrm{C}$ for $30 \mathrm{~min}$, followed by deparaffinization and rehydration using a graded series of xylene and ethanol. The slides were then heated in $0.01 \mathrm{M}$ citrate buffer for $20 \mathrm{~min}$ in a microwave oven, and endogenous peroxidase was blocked with hydrogen peroxide containing $0.3 \%$ methanol for $15 \mathrm{~min}$ at room temperature. Subsequently, the sections were placed in a humidified chamber and incubated for 30 min with $10 \%$ goat serum to block non-specific binding. Afterward, the sections were incubated overnight at $4^{\circ} \mathrm{C}$ with a monoclonal anti-RACK1 antibody at a dilution of 1:100 according to the manufacturer's specifications. Primary antibodies were detected using a biotinylated anti-goat IgG antibody diluted 1:200 followed by SP for $30 \mathrm{~min}$ at room temperature. Afterward, the immunoreaction was visualized using the Liquid DAB-Plus substrate kit (Zymed Laboratories Inc., San Francisco, CA, USA) according to the manufacturer's specifications. Finally, the sections were rinsed for $10 \mathrm{~min}$ in running tap water, counterstained with hematoxylin staining solution for $5 \mathrm{~min}$, dehydrated and mounted.

Co-immunoprecipitation. For co-immunoprecipitation analysis, cells were lysed with lysis buffer, and then the lysates were clarified by centrifugation at $12,000 \mathrm{rpm}$ for $10 \mathrm{~min}$ at $4^{\circ} \mathrm{C}$. The supernatants were mixed with the desired primary antibody overnight at $4^{\circ} \mathrm{C}$. After centrifugation, the immune complexes were captured by mixing with anti-Flag M2 agarose (Sigma) plus protein G-Sepharose (GE Healthcare, Buckinghamshire, $\mathrm{UK})$ for $2 \mathrm{~h}$ at $4^{\circ} \mathrm{C}$ in a rotator. When using the anti-RACK1 antibody in the co-immunoprecipitation assays, rabbit antimouse IgM was included as the bridging antibody. The beads were subsequently washed three times with washing buffer, eluted by boiling in 2X SDS loading buffer, and analyzed by SDS-PAGE and immunoblotting.

Immunofluorescence microscopy. HepG2 and MHCC97 cells on chamber slides were treated with $10 \mu \mathrm{g} / \mathrm{ml}$ tunicamycin (TM) for $4 \mathrm{~h}$. The slides were washed twice with PBS. The cells were fixed for $5 \mathrm{~min}$ in $4 \%$ paraformaldehyde and permeabilized for 2 min with $0.1 \%$ Triton X-100 in PBS. After blocking the cells with 5\% donkey serum albumin, the cells were incubated with a rabbit monoclonal RACK1 antibody at room temperature for $1 \mathrm{~h}$ and washed three times with PBS. Donkey anti-rabbit secondary antibodies were incubated with the cells for $45 \mathrm{~min}$, and the cells were washed three times with PBS. Immunofluorescence staining was examined under confocal microscopy (LSM 700; Zeiss), and images were processed using Volocity software (Perkin-Elmer, Waltham, MA, USA).

Quantitative real-time PCR analysis. Total RNA was extracted from frozen tissues and cell lines with TRIzol reagent (Invitrogen, Carlsbad, CA, USA) according to the manufacturer's instructions. After reverse transcription by M-MLV reverse transcriptase (Takara, Shiga, Japan), quantitative realtime PCR (qRT-PCR) was performed using the SYBR Premix Ex Taq (Takara) in the StepOne real-time PCR system (Applied Biosystems, Foster City, CA, USA). qRT-PCR was performed at $95^{\circ} \mathrm{C}$ for an initial $5 \mathrm{~min}$ followed by 30 cycles of denaturation at $95^{\circ} \mathrm{C}$ for $30 \mathrm{sec}$, annealing at $55^{\circ} \mathrm{C}$ for $30 \mathrm{sec}$ and extension at $72^{\circ} \mathrm{C}$ for $7 \mathrm{~min}$. Primer pairs for the target genes were as follows: RACK1, forward 5'-TTCTCCTCTGA CAACCGGCA-3' and reverse 5'-GCCATCCTTGCCTCCA GAA-3'; XBP1s, forward 5'-TGCTGAGTCCGCAGCAGG TG-3' and reverse 5'-GCTGGCAGGCTCTGGGGAAG-3'; and GAPDH, forward 5'-CGGAGTCAACGGATTTGGTC GTAT-3' and reverse 5'-AGCCTTCTCCATGGTGGTGAAG AC-3'. Data analysis was performed using the $\Delta \Delta \mathrm{Ct}$ method. Expression of the genes of interest was normalized to the expression of GAPDH.

Evaluation of cell apoptosis. The number of apoptotic cells was determined by Annexin V-FITC and PI staining. Briefly, the cells were collected, washed with PBS and incubated with Annexin V-FITC and PI according to the manufacturer's instructions (BD Biosciences, San Jose, CA, USA). Stained cells were analyzed using flow cytometry, and the assays were performed in triplicate.

Statistical analysis. The data are expressed as the mean \pm SD and were subjected to statistical analysis using SPSS version 17.0 (SPSS Inc., Chicago, IL, USA). Differences between groups were evaluated using a two-tailed Student's t-test or one-way ANOVA, and $\mathrm{P}<0.05$ was considered to indicate a statistically significant result.

\section{Results}

Activation of UPR and high expression of RACK1 are involved in HCC. Activated UPR and upregulated ER chaperones, such as IRE1 and GRP78, are found in a variety of cancer cells. Therefore, we first examined the expression of IRE1 and GRP78 via western blotting. As shown in Fig. 1A, the expression of IRE1 and GRP78 in the HCC cell lines was much higher than that in the human hepatic HL-7702 cell line. In addition, the expression of pIRE1 was higher in the HCC samples compared with that in their matched NT tissues (Fig. 1B). To determine whether RACK1 is involved in HCC, the relative mRNA and protein expression of RACK1 was analyzed using qRT-PCR and western blotting. As shown in Fig. 1A and B, the HCC cell lines showed high expression of RACK1 at both the mRNA and protein levels. Furthermore, the RACK1 protein level in the clinical HCC tissues was evaluated using immunohistochemistry. The expression of 

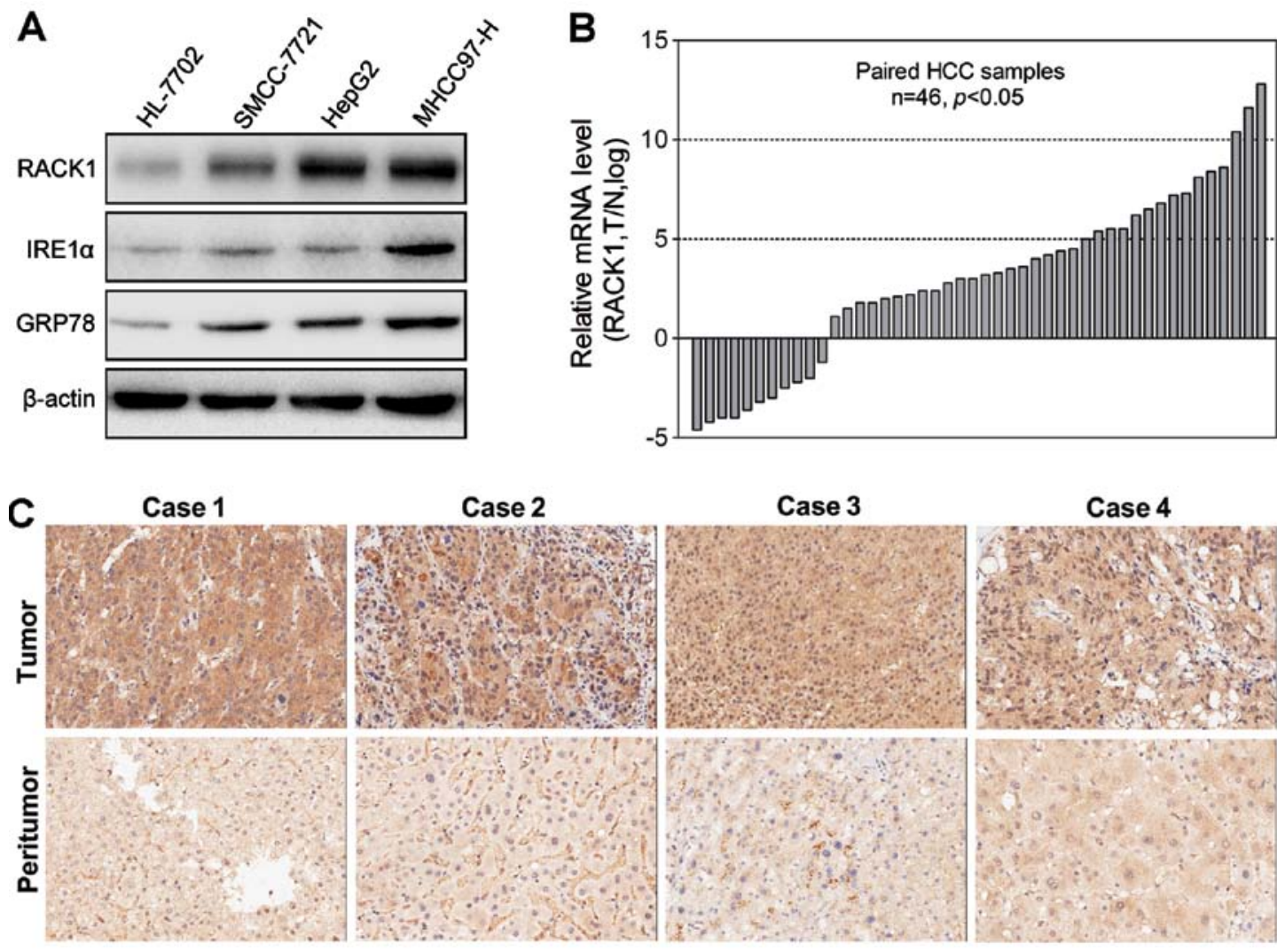

Figure 1. Expression of ER molecules and RACK1 in HCC. (A) Whole-cell extracts obtained from normal human hepatocytes HL7702 and HCC cell lines were evaluated using western blotting for RACK1, IRE1 and GRP78 expression. (B) RACK1 mRNA levels in HCC tissue specimens. Total RNA (2 $\mu \mathrm{g}$ ) was extracted from paired tumoral (tumor) and non-tumoral (adjacent) liver specimens, and the RACK1 mRNA level was analyzed using semi-quantitative reverse transcription-PCR with human RACK1 primers. GAPDH PCR products were run in parallel to confirm that equivalent amounts of cDNA were amplified. (C) RACK1 expression in HCC tissue specimens (immunohistochemical staining, magnification, x200).

RACK1 in the clinical HCC tissues was higher than that in the matched peritumoral liver tissues (Fig. 1C). Moreover, RACK1 expression was well correlated with the clinical stage of HCC (Table II). These results indicate that IRE1 and RACK1 are frequently upregulated in HCC, suggesting that activated UPR and highly expressed RACK1 may contribute to the tumorigenesis and progression during HCC.

Sorafenib induces ER stress-related apoptosis in HCC. We treated human hepatoma HepG2 cells with sorafenib at varying doses. The induction of apoptosis was evaluated using flow cytometry. To determine the activation status of IRE1 after treatment with sorafenib, the protein expression of pIRE1 and XBP1s was measured via western blotting. Our results showed that exposure to $2.5,5,10,15$ and $20 \mu \mathrm{M}$ sorafenib for $24 \mathrm{~h}$ increased cellular apoptosis in a dose-dependent manner compared with the levels observed in the standard nonstimulated cells (Fig. 2A). Additionally, sorafenib induced the upregulation of pIRE1 and XBP1s in hepatoma cells (Fig. 2B). Notably, the expression of pIRE1 and XBP1s did not increase progressively. Following low-dose $(<10 \mu \mathrm{M})$ treatment with sorafenib, the expression of pIRE1 and XBP1s increased progressively until reaching a peak and then declined after high-dose or prolonged treatment with sorafenib. IRE1 signaling activity is a key step in determining cell survival after ER stress. To understand the role of IRE1 signaling in sorafenib-induced apoptosis, HepG2 and MHCC97 cells
Table II. Correlation of RACK1 expression in HCC tissues with clinicopathological features of the 46 HCC patients.

\begin{tabular}{lrrrrr}
\hline & \multicolumn{4}{c}{ Intensity of staining } & \\
\cline { 3 - 5 } Features & $\mathrm{N}$ & 1 & 2 & 3 & P-value \\
\hline Age (years) & & & & & 0.668 \\
$\quad 660$ & 35 & 9 & 15 & 11 & \\
$>60$ & 11 & 3 & 3 & 5 & \\
Gender & & & & & 1.000 \\
$\quad$ Male & 40 & 8 & 18 & 14 & \\
Female & 6 & 1 & 3 & 2 & \\
Tumor size (cm) & & & & & 0.008 \\
$\quad 55$ & 24 & 7 & 12 & 5 & \\
$>5$ & 22 & 3 & 4 & 15 & \\
Clinical stage & & & & & 0.028 \\
I-II & 16 & 5 & 5 & 6 & \\
III-IV & 30 & 3 & 6 & 21 & \\
\hline
\end{tabular}

were transfected with siIRE1 and then exposed to sorafenib. The flow cytometric analysis of apoptosis revealed that IRE1 siRNA-transfected cells exhibited significantly increased cell death compared with the control cells (Fig. 2C). These results 
A
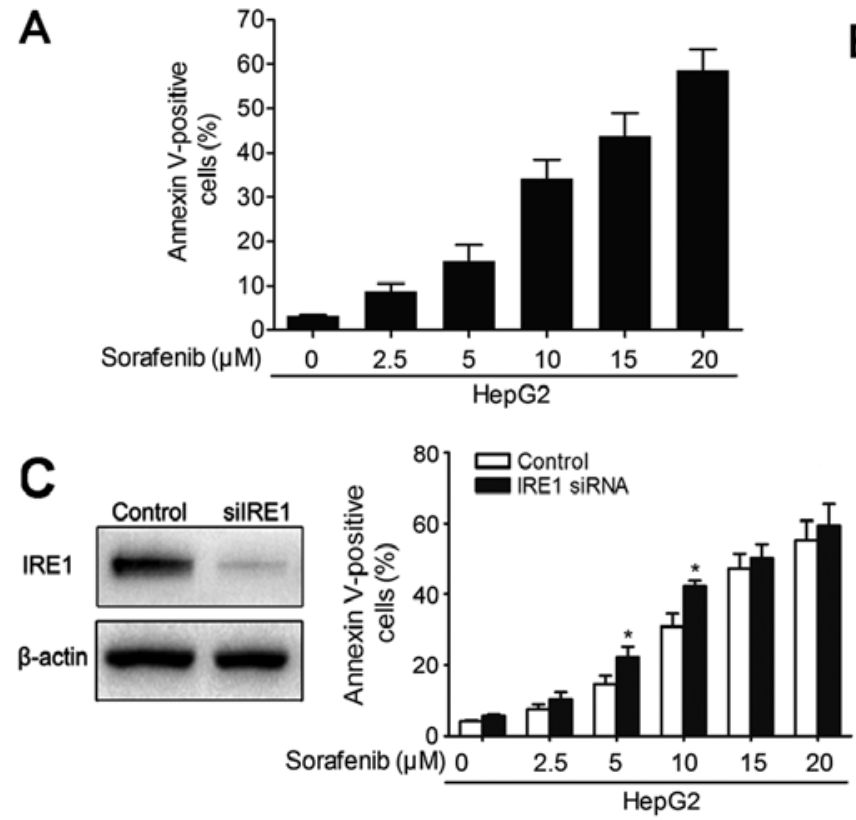

B
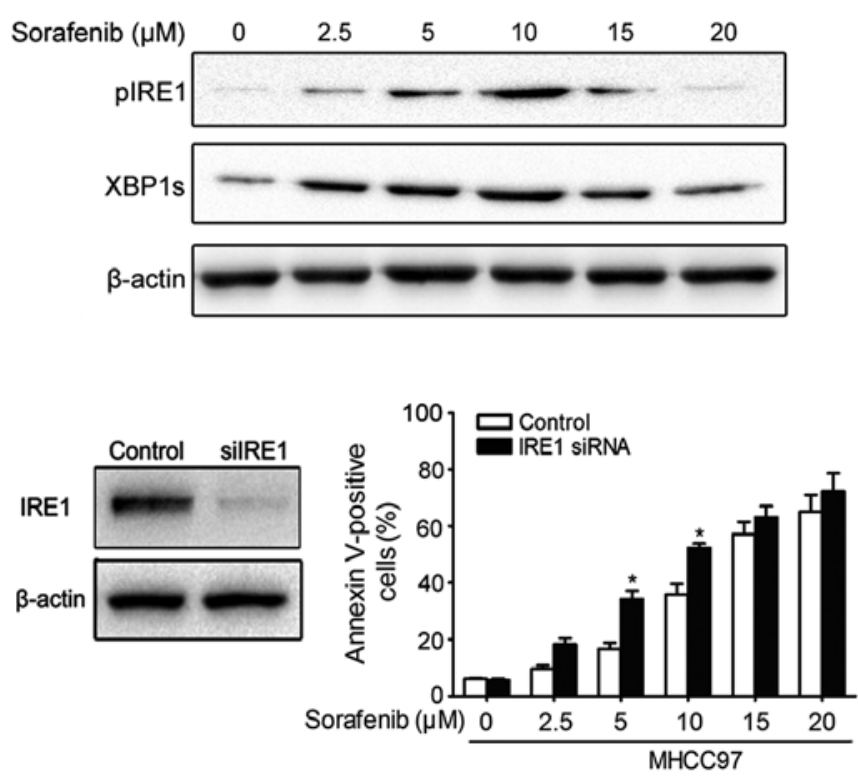

Figure 2. Sorafenib induces ER stress in HCC cells. (A) HepG2 cells were treated for $24 \mathrm{~h}$ with $0,2.5,5,10,15$ or $20 \mu \mathrm{M}$ sorafenib. Then, cellular apoptosis was evaluated by Annexin V staining, and (B) the expression of IRE1 and XBP1s was detected by western blotting. (C) HCC cell lines, HepG2 and MHCC97, were infected with siIRE1 or control siRNA for $48 \mathrm{~h}$, and then treated with sorafenib at the indicated dose for $24 \mathrm{~h}$. Cell apoptosis was evaluated by Annexin V staining $\left({ }^{*} \mathrm{P}<0.05\right.$ vs. the control).
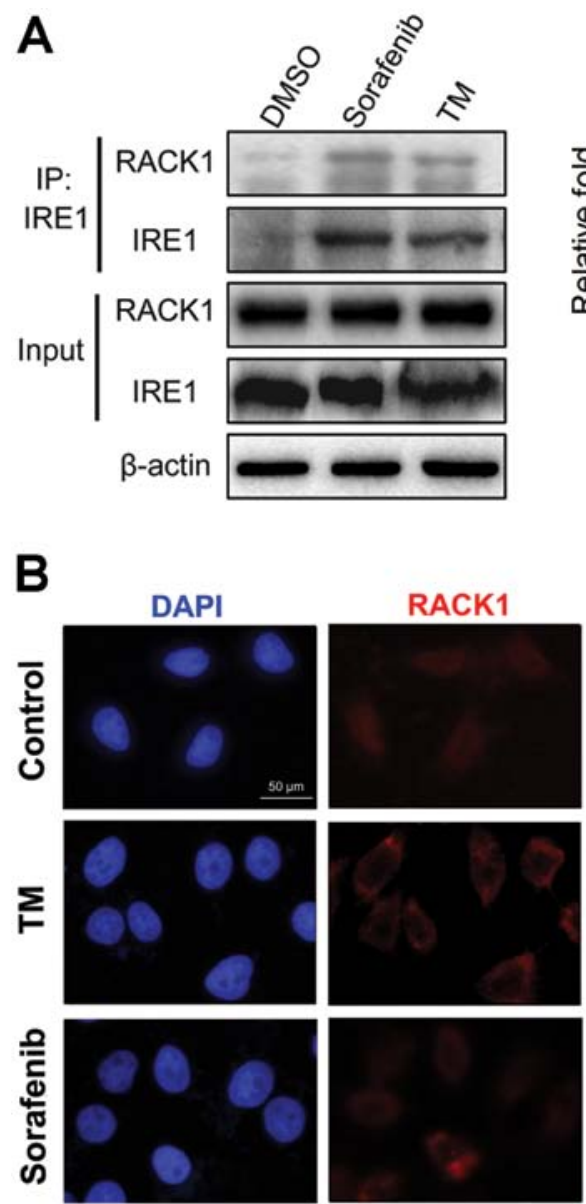
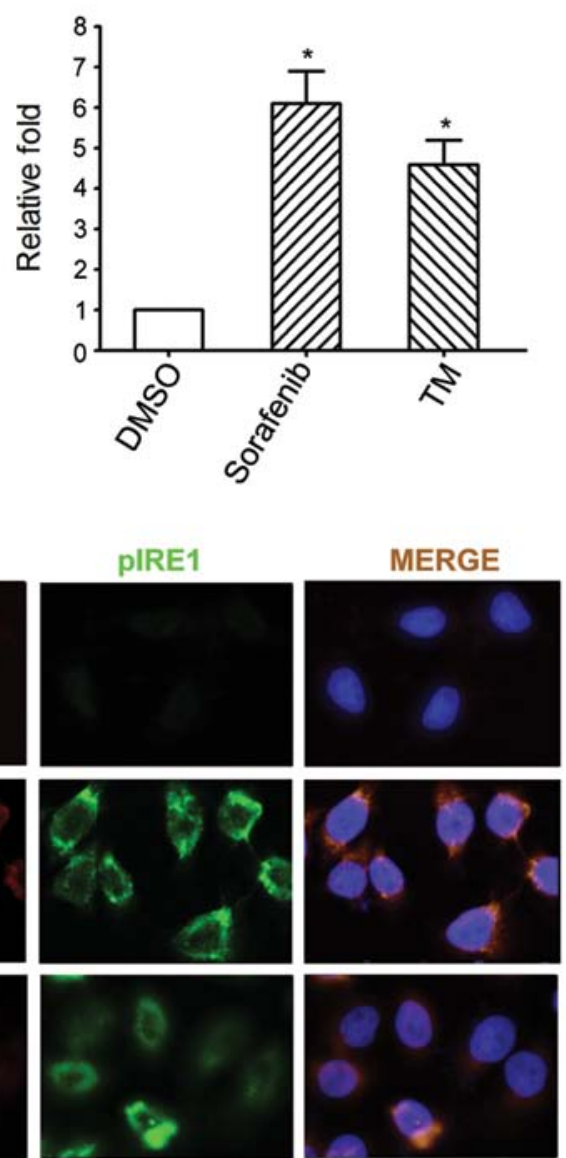

Figure 3. RACK1 interacts with IRE1 in sorafenib-treated or ER stress-induced HCC cells. (A) HepG2 cells were treated with DMSO, TM (10 $\mu \mathrm{g} / \mathrm{ml})$ or sorafenib $(10 \mu \mathrm{M})$ for $24 \mathrm{~h}$. The HCC cells were then harvested, and immunoprecipitation was performed with an anti-RACK1 antibody. An antibody against IRE1 was used for immunoblotting ("P $<0.05$ vs. cells treated with DMSO). (B) IRE1 aggregated and co-localized with RACK1 in the cytoplasm after treatment with TM and sorafenib. HepG2 cells were treated with sorafenib or TM. The cells were stained using antibodies specific for IRE1 (red) or RACK1 (green). Scale bar, $50 \mu \mathrm{m}$. 
A
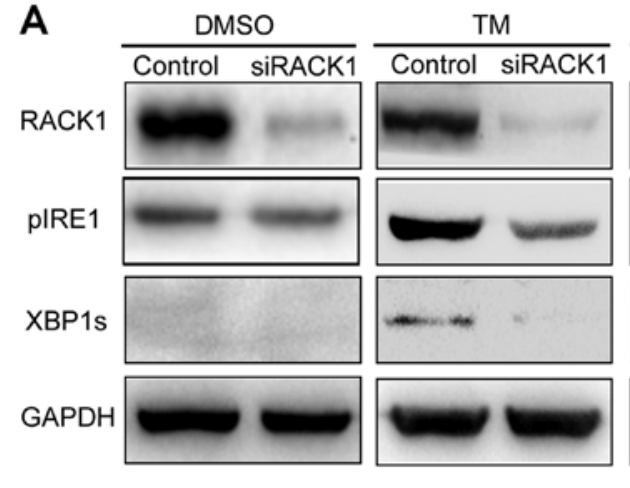

Sorafenib

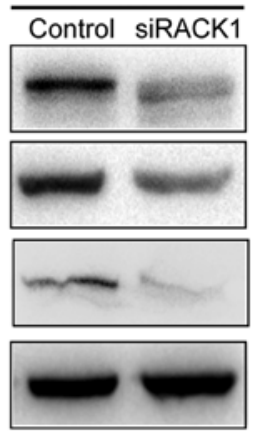

B

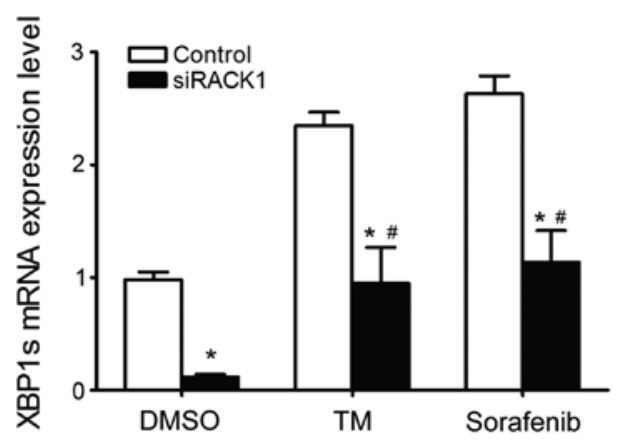

Figure 4. The effect of RACK1 on the activation of the IRE1/XBP1 axis. Forty-eight hours after transfection of HepG2 cells with RACK1 siRNA or control non-targeting siRNA, the cells were treated with DMSO, TM $(10 \mu \mathrm{g} / \mathrm{ml})$ or sorafenib $(10 \mu \mathrm{M})$ for $24 \mathrm{~h}$. (A) The protein levels of pIRE1 and XBP1s were evaluated using western blotting, and (B) the mRNA level of XBP1s was evaluated using qRT-PCR. ("P $<0.05$ vs. the control, ${ }^{\prime} \mathrm{P}<0.05$ vs. siRACK1 cells treated with DMSO).

indicate that sorafenib induces ER stress-related apoptosis in HCC, and activation of the IRE1/XBP1 signaling pathway may play a protective role when HCC cells encounter ER stress in response to sorafenib.

RACK1 interacts with IRE1 in sorafenib-stimulated HCC cells. Previous studies identified an interaction between IRE1 and RACK1 in human embryonic kidney (HEK) 293 T cells (31). To determine the interaction of IRE1 and RACK1 under physiological or ER stress conditions, we performed immunoprecipitation in HepG2 cells treated with TM or sorafenib. TM is a well-known ER stressor that acts by inhibiting protein $\mathrm{N}$ glycosylation (33). As shown in Fig. 3A, an increased level of RACK1 was found in the IRE1 immunoprecipitates from the TM- or sorafenib-treated cells compared with the level observed in the control cells. To visualize whether IRE1 and RACK1 co-localize in the cytoplasm, we examined the subcellular localization of IRE1 relative to RACK1 in the TM- or sorafenib-treated cells using double immunofluorescence staining. As shown in Fig. 3B, IRE1 aggregated and co-localized with RACK1 in the cytoplasm after treatment with TM and sorafenib. In contrast, the co-localization of IRE1 aggregates with RACK1 was seldom found in the control cells.

RACK1 is essential for the activation of IRE1 signaling. To investigate whether the RACK1/IRE1 interaction affects activation of the UPR, RACK1 protein expression was knocked down with siRNA specific for RACK1. Western blot analysis revealed that siRACK1 significantly suppressed the basal level of RACK1. Knockdown of RACK1 suppressed the phosphorylation of IRE1 in response to TM or sorafenib compared with that of cells transfected with a scrambled negative control (Fig. 4A). Moreover, reduced pIRE1 expression under the condition of RACK1 knockdown was associated with decreased XBP1 mRNA splicing and XBP1s expression (Fig. 4B). These results indicate that RACK1 may exert distinct actions in the regulation of IRE1 signaling in response to ER stress.

RACK1 modulates sorafenib-induced apoptosis of HCC cells. Several reports have implicated IRE1 in pathways associated with apoptosis resistance. Since RACK1 is required for the activation of IRE1 signaling under ER stress, it appeared likely that RACK1 was also essential for the protective effect of the UPR. To test this hypothesis, HepG2 and MHCC97 cells were transfected with pGFP-RACK1 or si-RACK1 and then exposed to sorafenib. The apoptotic effects of sorafenib were determined using flow cytometric assays. The results demonstrated that the overexpression of RACK1 protected HCC cells from sorafenib-induced apoptosis (Fig. 5A, left histograms). In contrast, we observed that sorafenib induced significant apoptosis in HCC cells transfected with si-RACK1 (Fig. 5B, left histograms). As XBP1 splicing is characteristic of UPR activation, we next evaluated the levels of the spliced form of XBP1 using qRT-PCR. At the mRNA level, XBP1s was significantly upregulated in RACK1 gain-of-function cells (Fig. 5A, right histograms). However, XBP1s was reduced in the RACK1 knockdown cells (Fig. 5B, right histograms). These findings indicate that RACK1 is involved in the protective effect of UPR against sorafenib-induced apoptosis.

\section{Discussion}

Sorafenib is one of the preferred systemic therapies in the treatment of advanced HCC. However, little is known concerning the mechanism by which sorafenib induces cell death and the drug resistance gained in response to HCC treatment. Activation of the UPR by the IRE1 signaling pathway is a key component of cell survival decisions in response to ER stress (34). We observed the activation of IRE1 in the sorafenib-treated HCC cells, thereby indicating that sorafenib induces the UPR and ER stress-related apoptosis. The combination of sorafenib and an agent that targets the UPR is a promising therapeutic strategy for the treatment of HCC.

RACK1, a multifaceted scaffolding protein, participates in various signaling pathways and plays an important role in multiple cellular functions. RACK1 has been associated with the migration and proliferation of various types of tumors, including HCC. Consistent with recent reports, our findings showed that RACK1 is highly expressed in HCC tissues and cell lines, indicating that RACK1 may be involved in the tumorigenesis and prognosis of HCC. We discovered that the levels of RACK1 and IRE1 were markedly increased in the sorafenib-treated HCC cells, and we also indicated that RACK1 interacts with IRE1 intercellularly and that the two are co-localized in the cytoplasm by immunoprecipation and immunofluorescence. Knockdown of RACK1 expression 

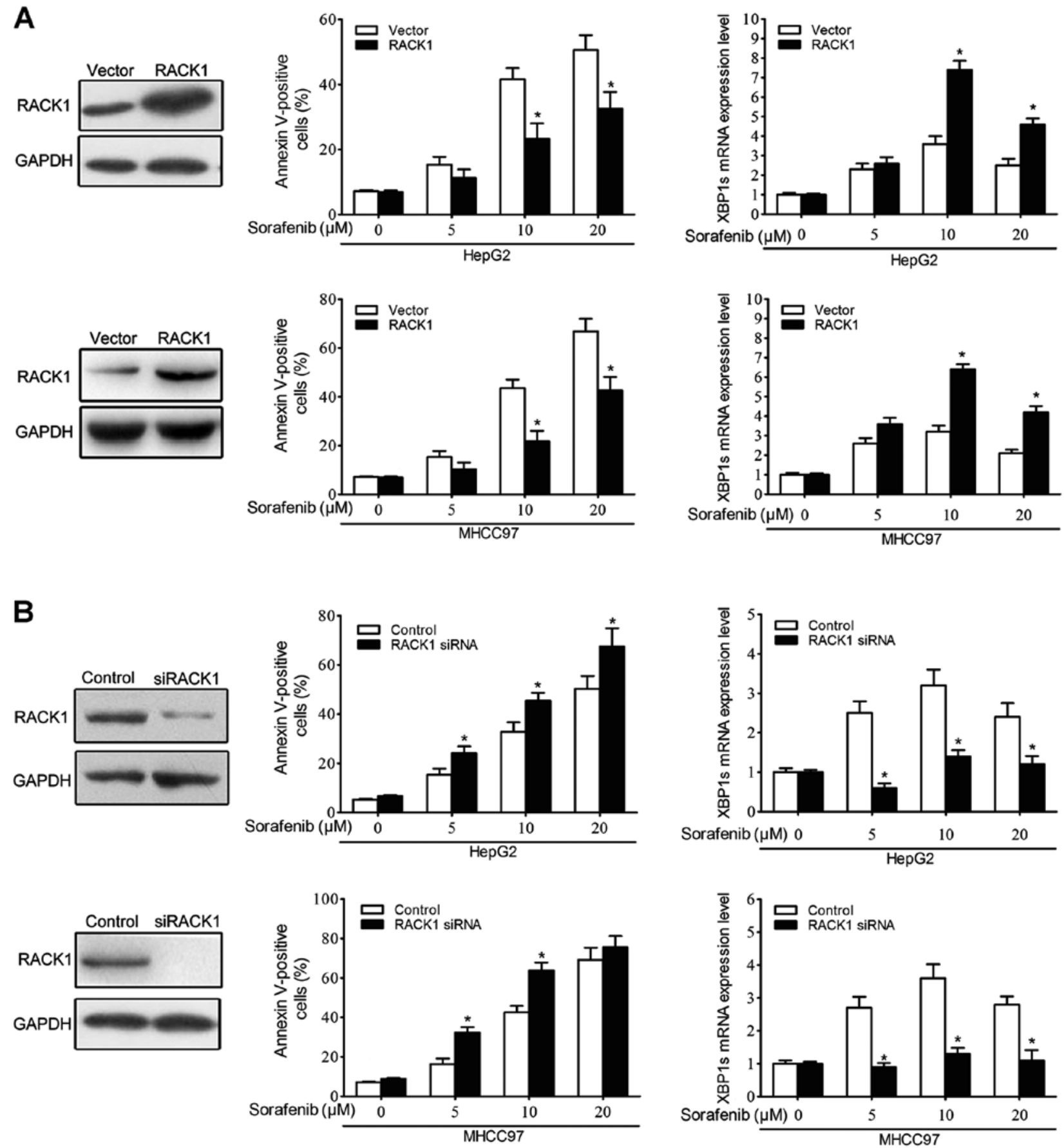

Figure 5. RACK1 protects HCC cells from apoptosis induced by sorafenib. Following a 48-h transfection with wild-type RACK1, RACK1 siRNA plasmid or control, HepG2 and MHCC97 cells were treated with sorafenib at the indicated doses $(5,10$ and $20 \mu \mathrm{M})$ for $24 \mathrm{~h}$. Cell apoptosis was determined by Annexin V staining. The cell lysates were analyzed by immunoblotting and qRT-PCR. (A) The overexpression of RACK1 promoted XBP1 gene expression and enhanced sorafenib resistance in the HCC cell lines. (B) RACK1 depletion suppressed XBP1 gene expression and increased the sensitivity of HCC cells to sorafenibinduced apoptosis ( $\mathrm{P}<0.05$ vs. control).

severely repressed the phosphorylation of IRE1 in response to TM or sorafenib and led to inhibition of the UPR. These results raise the possibility that RACK1 and IRE1 work as a complex to mediate activation of the IRE1 signaling pathway during sorafenib-induced UPR, thereby modulating the ER stressinduced apoptosis of HCC cells after exposure to sorafenib.

Activation of the UPR is an adaptive response that allows cells to survive prolonged ER stress conditions in various solid tumors (35). The IRE1 signaling pathway functions as a switch between cytoprotective and proapoptotic outcomes in response to ER stress, and the activation of IRE1 plays a crucial role in the UPR to enhance cell survival (34). Our results indicate that sorafenib treatment triggers the UPR and ER stress-related apoptosis in HCC cells, given the essential role of IRE1 activation in governing the switch from adaptive UPR to ER stress-associated apoptosis in sorafenib-treated HCC cells. It is important to understand how RACK1 affects the actions of other IRE1 interactors, such as XBP1, CHOP and Bcl-2. Activated IRE1 can function as a transmembrane kinase to control the splicing of XBP1 mRNA. The activation 
of IRE1 in various temporal patterns during ER stress influences the cell's ultimate fate, whereas XBP1 mRNA splicing is only activated during the adaptive phase of ER stress. Previous reports indicate that XBP1 expression is increased in certain solid tumors in association with chemotherapy resistance $(36,37)$. In the present study, we found that sorafenib was able to stimulate the UPR in HCC cells. A low-dose and short treatment with sorafenib was able to enhance the expression of IRE1 and XBP1 mRNA splicing, which may play a protective role and improve cell survival. We also observed that when cells encounter severe or prolonged stress, IRE1 becomes constitutively active while XBP1 mRNA splicing is downregulated. When the expression of IRE1 was knocked down using siIRE1, XBP1 mRNA splicing decreased, and IRE1 siRNA-transfected cells exhibited a significant increase in cell death following sorafenib treatment. These results indicate that activation of the UPR protects cells from ER stress induced by sorafenib, and targeting the IRE1/XBP1 axis is a promising antitumor strategy.

Recent studies $(27,29)$ have demonstrated that RACK1 plays a context-dependent role in tumorigenesis and is responsible for stress-mediated chemotherapy resistance. Our results demonstrated that RACK1 modulated sorafenib-induced apoptosis by regulating IRE1 activity, and the overexpression of RACK1 promoted the phosphorylation of IRE1 and inhibited apoptosis induced by sorafenib. In contrast, knockdown of RACK1 significantly enhanced the lethality of sorafenib to HCC cells. These results raise the possibility that RACK1 forms a complex with IRE1 following treatment with sorafenib, and may influence the activation state of the IRE1/XBP1 axis, thereby alleviating the ER stress induced by sorafenib. Thus, we hypothesize that RACK1 may be a potential molecular targeted cancer treatment.

Taken together, the present study confirmed that activation of the IRE1 signaling pathway is involved in sorafenib-induced UPR. Our data raise the possibility that the RACK1/IRE1 complex might contribute to activation of the UPR in HCC cells. Targeting RACK1 is a potential adjuvant therapeutic strategy in combination with sorafenib for advanced HCC.

\section{Acknowledgements}

The present study is supported by grants from the Natural Science Foundation of China (nos. 81070363, 81030010 and 81101818).

\section{References}

1. Ferlay J ISME: GLOBOCAN 2012: Estimated Cancer Incidence, Mortality and Prevalence Worldwide in 2012. http://globocan.iarc. fr/Default.aspx. IARC CancerBase, 2013.

2. Flores A and Marrero JA: Emerging trends in hepatocellular carcinoma: Focus on diagnosis and therapeutics. Clin Med Insights Oncol 8: 71-76, 2014.

3. Peck-Radosavljevic M: Drug therapy for advanced-stage liver cancer. Liver Cancer 3: 125-131, 2014.

4. Llovet JM, Ricci S, Mazzaferro V, Hilgard P, Gane E, Blanc JF, de Oliveira AC, Santoro A, Raoul JL, Forner A, et al; SHARP Investigators Study Group: Sorafenib in advanced hepatocellular carcinoma. N Engl J Med 359: 378-390, 2008.

5. Gauthier A and Ho M: Role of sorafenib in the treatment of advanced hepatocellular carcinoma: An update. Hepatol Res 43 : $147-154,2013$
6. Wilhelm SM, Carter C, Tang L, Wilkie D, McNabola A, Rong H, Chen C, Zhang X, Vincent P, McHugh M, et al: BAY 43-9006 exhibits broad spectrum oral antitumor activity and targets the RAF/MEK/ERK pathway and receptor tyrosine kinases involved in tumor progression and angiogenesis. Cancer Res 64: 7099-7109, 2004

7. Liu L, Cao Y, Chen C, Zhang X, McNabola A, Wilkie D, Wilhelm S, Lynch M and Carter C: Sorafenib blocks the RAF/ MEK/ERK pathway, inhibits tumor angiogenesis, and induces tumor cell apoptosis in hepatocellular carcinoma model PLC/ PRF/5. Cancer Res 66: 11851-11858, 2006.

8. Rahmani M, Davis EM, Bauer C, Dent P and Grant S: Apoptosis induced by the kinase inhibitor BAY 43-9006 in human leukemia cells involves down-regulation of Mcl-1 through inhibition of translation. J Biol Chem 280: 35217-35227, 2005.

9. Wang WA, Groenendyk J and Michalak M: Endoplasmic reticulum stress associated responses in cancer. Biochim Biophys Acta 1843: 2143-2149, 2014.

10. Li X, Zhang $\mathrm{K}$ and $\mathrm{Li} \mathrm{Z}$ : Unfolded protein response in cancer: The physician's perspective. J Hematol Oncol 4: 8, 2011.

11. Vandewynckel YP,Laukens D, Geerts A, Bogaerts E, Paridaens A, Verhelst X, Janssens S, Heindryckx F and Van Vlierberghe H: The paradox of the unfolded protein response in cancer. Anticancer Res 33: 4683-4694, 2013.

12. Zhang XD, Hersey P, Tay KH, Tseng H, Jiang CC and Dong L: Adaptation to ER stress as a mechanism of resistance of melanoma to treatment. In: Current Management of Malignant Melanoma, Cao M. (ed). InTech, Rijeka, Croatia, 2011.

13. Suh DH, Kim MK, Kim HS, Chung HH and Song YS: Unfolded protein response to autophagy as a promising druggable target for anticancer therapy. Ann NY Acad Sci 1271: 20-32, 2012.

14. Gardner BM and Walter P: Unfolded proteins are Ire1-activating ligands that directly induce the unfolded protein response. Science 333: 1891-1894, 2011.

15. Schröder M and Kaufman RJ: ER stress and the unfolded protein response. Mutat Res 569: 29-63, 2005.

16. Han D, Lerner AG, Vande Walle L, Upton JP, Xu W, Hagen A, Backes BJ, Oakes SA and Papa FR: IRElalpha kinase activation modes control alternate endoribonuclease outputs to determine divergent cell fates. Cell 138: 562-575, 2009.

17. Hassler J, Cao SS and Kaufman RJ: IRE1, a double-edged sword in pre-miRNA slicing and cell death. Dev Cell 23: 921-923, 2012.

18. Tirosh B, Iwakoshi NN, Glimcher LH and Ploegh HL: Rapid turnover of unspliced Xbp-1 as a factor that modulates the unfolded protein response. J Biol Chem 281: 5852-5860, 2006.

19. Yoshida H, Matsui T, Yamamoto A, Okada T and Mori K: XBP1 mRNA is induced by ATF6 and spliced by IRE1 in response to ER stress to produce a highly active transcription factor. Cell 107: 881-891, 2001.

20. Maurel M, Chevet E, Tavernier J and Gerlo S: Getting RIDD of RNA: IRE1 in cell fate regulation. Trends Biochem Sci 39: 245-254, 2014.

21. Chitnis N, Pytel D and Diehl JA: UPR-inducible miRNAs contribute to stressful situations. Trends Biochem Sci 38: 447-452, 2013.

22. Lin JH, Li H, Yasumura D, Cohen HR, Zhang C, Panning B, Shokat KM, Lavail MM and Walter P: IRE1 signaling affects cell fate during the unfolded protein response. Science 318: 944-949, 2007.

23. Ron D, Chen CH, Caldwell J, Jamieson L, Orr E and MochlyRosen D: Cloning of an intracellular receptor for protein kinase C: A homolog of the beta subunit of $G$ proteins. Proc Natl Acad Sci USA 91: 839-843, 1994.

24. Adams DR, Ron D and Kiely PA: RACK1, A multifaceted scaffolding protein: Structure and function. Cell Commun Signal 9: 22, 2011.

25. Ma J, Wu R, Zhang Q, Wu JB, Lou J, Zheng Z, Ding JQ and Yuan Z: DJ-1 interacts with RACK1 and protects neurons from oxidative-stress-induced apoptosis. Biochem J 462: 489-497, 2014.

26. McCahill A, Warwicker J, Bolger GB, Houslay MD and Yarwood SJ: The RACK1 scaffold protein: A dynamic cog in cell response mechanisms. Mol Pharmacol 62: 1261-1273, 2002.

27. Wu J, Meng J, Du Y, Huang Y, Jin Y, Zhang J, Wang B, Zhang Y, Sun $M$ and Tang J: RACK1 promotes the proliferation, migration and invasion capacity of mouse hepatocellular carcinoma cell line in vitro probably by PI3K/Racl signaling pathway. Biomed Pharmacother 67: 313-319, 2013. 
28. Cao XX, Xu JD, Liu XL, Xu JW, Wang WJ, Li QQ, Chen Q, Xu ZD and Liu XP: RACK1: A superior independent predictor for poor clinical outcome in breast cancer. Int J Cancer 127: 1172-1179, 2010.

29. Ruan Y, Sun L, Hao Y, Wang L, Xu J, Zhang W, Xie J, Guo L, Zhou L, Yun X, et al: Ribosomal RACK1 promotes chemoresistance and growth in human hepatocellular carcinoma. J Clin Invest 122: 2554-2566, 2012.

30. Arimoto K, Fukuda H, Imajoh-Ohmi S, Saito H and Takekawa M: Formation of stress granules inhibits apoptosis by suppressing stress-responsive MAPK pathways. Nat Cell Biol 10: 1324-1332, 2008.

31. Qiu Y, Mao T, Zhang Y, Shao M, You J, Ding Q, Chen Y, Wu D, Xie D, Lin X, et al: A crucial role for RACK1 in the regulation of glucose-stimulated IRElalpha activation in pancreatic beta cells. Sci Signal 3: ra7, 2010.

32. Liver Cancer Study Group of Japan: The General Rules for the Clinical and Pathological Study of Primary Liver Cancer. 2nd English edition. Kanehara \& Co., Ltd., Tokyo, pp13-14, 2003.
33. Rojas R, Segovia C, Trombert AN, Santander J and Manque P: The effect of tunicamycin on the glucose uptake, growth, and cellular adhesion in the protozoan parasite Crithidia fasciculata. Curr Microbiol 69: 541-548, 2014.

34. Chen Y and Brandizzi F: IRE1: ER stress sensor and cell fate executor. Trends Cell Biol 23: 547-555, 2013.

35. Li X, Zhang K and Li Z: Unfolded protein response in cancer: The physician's perspective. J Hematol Oncol 4: 8, 2011.

36. Fujimoto T, Onda M, Nagai H, Nagahata T, Ogawa K and Emi M: Upregulation and overexpression of human X-box binding protein 1 (hXBP-1) gene in primary breast cancers. Breast Cancer 10: 301-306, 2003.

37. Shuda M, Kondoh N, Imazeki N, Tanaka K, Okada T, Mori K, Hada A, Arai M, Wakatsuki T, Matsubara O, et al: Activation of the ATF6, XBP1 and grp78 genes in human hepatocellular carcinoma: A possible involvement of the ER stress pathway in hepatocarcinogenesis. J Hepatol 38: 605-614, 2003. 